BMJ Open

Diabetes

Research

\& Care

\section{Algorithms for personalized therapy of type 2 diabetes: results of a web-based international survey}

To cite: Gallo M, Mannucci E, De Cosmo S, et al. Algorithms for personalized therapy of type 2 diabetes: results of a web-based international survey. BMJ Open Diabetes Research and Care 2015;3:e000109. doi:10.1136/bmjdrc-2015000109

Received 12 April 2015 Revised 17 June 2015 Accepted 12 July 2015

\section{CrossMark}

For numbered affiliations see end of article.

Correspondence to

Dr Marco Gallo;

mgall04@cittadellasalute.to.it

\section{ABSTRACT}

Objective: In recent years increasing interest in the issue of treatment personalization for type 2 diabetes (T2DM) has emerged. This international web-based survey aimed to evaluate opinions of physicians about tailored therapeutic algorithms developed by the Italian Association of Diabetologists (AMD) and available online, and to get suggestions for future developments. Another aim of this initiative was to assess whether the online advertising and the survey would have increased the global visibility of the AMD algorithms.

Research design and methods: The web-based survey, which comprised five questions, has been available from the homepage of the web-version of the journal Diabetes Care throughout the month of December 2013, and on the AMD website between December 2013 and September 2014. Participation was totally free and responders were anonymous.

Results: Overall, 452 physicians ( $M=58.4 \%)$ participated in the survey. Diabetologists accounted for $76.8 \%$ of responders. The results of the survey show wide agreement $(>90 \%)$ by participants on the utility of the algorithms proposed, even if they do not cover all possible needs of patients with T2DM for a personalized therapeutic approach. In the online survey period and in the months after its conclusion, a relevant and durable increase in the number of unique users who visited the websites was registered, compared to the period preceding the survey.

Conclusions: Patients with T2DM are heterogeneous, and there is interest toward accessible and easy to use personalized therapeutic algorithms. Responders opinions probably reflect the peculiar organization of diabetes care in each country.

\section{INTRODUCTION}

In recent years, the increased availability of pharmacological options has made the therapeutic management of type 2 diabetes (T2DM) more complex and controversial, prompting various scientific societies to enact their own algorithms and guidelines. ${ }^{1-3}$

\section{Key messages}

- There is a clinical need for personalized algorithms for patients with T2DM.

- This international survey aimed to evaluate opinions of physicians about tailored therapeutic algorithms developed by the Italian Association of Diabetologists.

- The Italian algorithm for the treatment of T2DM may improve the approach to a personalized management of T2DM.

It is widely recognized that treatment goals should be individualized on the basis of patients' characteristics. ${ }^{4}$ In addition, although it is difficult to establish an overall superiority of one agent over another in terms of safety and efficacy, the possibility exists that different drugs are more advisable in specific subgroups of patients. The concept of personalized management decisions has consequently spread, in diabetology as well as in other areas of medicine. Nowadays, several scientific societies emphasize the need for individualized and 'patientcentered' approaches. ${ }^{156}$ At the same time, a generic appeal to personalized therapy risks to become a justification of empiricism, unless some details are provided on the parameters leading to personalized choices. ${ }^{7}$

Since 2010, the Italian Association of Diabetologists (Associazione Medici Diabetologi, AMD) recognized the need to develop personalized treatment plans for people with T2DM. Among patients' characteristics identified as possible determinants of therapeutic choices, patients' age, presence of macrovascular diabetic complications or other comorbidities (ie, obesity, renal failure or disabling conditions), occupational risks potentially related to hypoglycemia, and the cognitive and nutritional 
status of the patient should be taken under active consideration. These algorithms conceptual framework is formed by the patients' phenotyping, the personalization of the objectives, a rational use of self-monitoring blood glucose, ${ }^{8}$ as well as by the choice of drugs based on phenotype and individual glycemic profiles. When choosing an antidiabetic over another, the decision is not guided only by criteria of effectiveness, but also by pharmacodynamic properties, safety profile and costs. ${ }^{9}{ }^{10}$ From the beginning, the decision to favor webbased over printed algorithms (borrowed from the example of the Finnish guidelines, which have been pioneer in this regard $)^{56}$ aimed to offer a simple interactive tool, easy to use in daily clinical practice from every PC connected to the web. AMD algorithms are available online as a browser operated interactive tool, in English and Italian. ${ }^{11}$ The reader can quickly locate the phenotype of interest according to the patient's clinical features, and easily follow a step-by-step suggested additive therapeutic pathway.

Aware that these algorithms must be continuously updated, AMD aims to acknowledge the latest data arising from the evolution of scientific knowledge. Nevertheless, the most important improvements can only come from the critical contribution of all the physicians who accept to test them in their daily clinical routine, indicating their strengths and limitations.

For this reason, in December 2013 AMD led an initiative of online advertising on the homepage of the web version of the journal Diabetes Care. Through banner advertising, readers from three continents (Europe, North America and Asia) were invited to visit the website of the algorithms, participating in an online survey of a few minutes. The main objective of this survey was to collect participants' opinions on the actual usefulness and critical issues of the algorithms, together with their suggestions for future developments. Another aim of this initiative was to assess whether the online advertising and the survey would have increased the global visibility of the website. This report presents the results of this initiative.

\section{MATERIALS AND METHODS}

The web-based survey launched by AMD has been available from the homepage of the web-version of the journal Diabetes Care (http://care.diabetesjournals.org) throughout the month of December 2013. Furthermore, the survey has been available on the AMD website (http://www.aemmedi.it) between December 2013 and September 2014. In Italy, this initiative was promoted through email communications to all AMD members and during the meetings of the scientific society. Participation was totally free and responders were anonymous. The survey included three sections:

1. A brief presentation of the AMD algorithms on the personalized therapy for T2DM, with a clarification on the rationale behind the proposals made and an invitation to visit the website.

The presentation included the main purpose of the online survey, that is, to know the participant opinion on the algorithms, with the aim of assessing their applicability in clinical practice and collecting suggestions for their implementation.

2. Characteristics of responders: gender, age, Country, specialty (ie, diabetologist/endocrinologist, general practitioner (GP), etc) and number of patients with T2DM seen in a typical month.

3. Participants' opinions about the general usefulness of algorithms dedicated to the treatment of diabetes, the conceptual structure of the AMD algorithms and their utility in the clinical practice.

In detail, five questions were posed to participants:

A. Do you think that computerized algorithms can be useful in your clinical practice? (yes often/yes sometimes/not at all)

B. Do you think that the six main subcategories of patients with T2DM identified by AMD algorithms are correct? (yes/no)

C. Do you think that the phenotypization of patients with T2DM according to the type and prevalence of their blood sugar levels (fasting, preprandial, and postprandial) can be relevant for choosing the most appropriate antidiabetic therapy? (yes/no/i don't know)

D. What did you like most of the AMD algorithms? (novelty/availability online/easy to use/completeness/other)

E. Do you think that the algorithms are a useful tool for: (GPs/diabetologist/endocrinologist/internists/ other specialties/none (multiple choice admitted))

\section{Statistical analysis}

Continuous data are expressed as mean $\pm \mathrm{SD}$.

A stepwise multivariate logistic regression was applied to identify physicians' characteristics independently associated with a positive opinion ('Yes sometimes' or 'Yes often' vs 'Not at all') on the usefulness of algorithms in clinical practice. Physician's age, gender, specialty, country of origin, and the number of patients with T2DM seen in a typical month were inputed as covariates.

All analyses were performed using SPSS V.20.0.

\section{RESULTS}

\section{Characteristics of responders}

Overall, 452 physicians $(\mathrm{M}=58.4 \%)$ participated in the initiative. Of those, $40.7 \%$ were aged $40-55$ years, and $48.5 \%$ were over 55 years. Diabetologists and endocrinologists accounted for $76.8 \%$ of responders, GPs for $8.4 \%$, whereas internists and other specialists for $14.8 \%$ (figure 1). The great majority of responders were from Italy $(85.6 \%)$, but 75 participants were from other countries (mainly from Europe and from the USA, but also from Far and Middle East Asia, Africa, Oceania, and the 


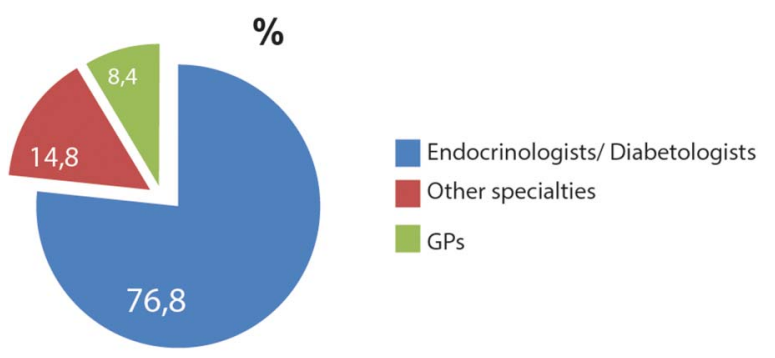

Figure 1 Total respondents ( $\mathrm{N}=452$ ) divided according to specialty practice, that is, diabetologists/endocrinologists $(N=347)$, other specialties $(n=67)$ or general practitioner $(n=38)$.

Caribbean). Most of the responders worked at a hospital $(79 \%)$, and $24.3 \%$ worked as private practitioners.

As to the number of type 2 patients with diabetes seen in a typical month, $51.6 \%$ of the 364 responders to this questions stated that they see 50-200 patients, whereas $45.6 \%$ see more than 200 patients with T2DM in a month.

\section{Responders opinions}

The algorithms were perceived as useful for one's own practice by $418(92.5 \%)$ of responders ('sometimes' for 219 (48.5\%) and 'often' for 199 (44\%); figure 2A). Responses about the algorithms were very positive irrespective of specialty, Country of origin, gender, age category or number of patients seen in a typical month of respondents.

Algorithms were considered useful for diabetes specialists, GPs, and internists by 297 (65.7\%), 254 (56.2\%) and $166(36.7 \%)$ of responders, respectively. Proportion of positive responses were not significantly different for age categories, gender or number of patients per month. Conversely, a significantly $(p<0.02)$ greater proportion of
Figure 2 (A-E) Participants answers to the questions of the online survey.
A Do you think that computerized algorithms can be useful to your clinical practice?

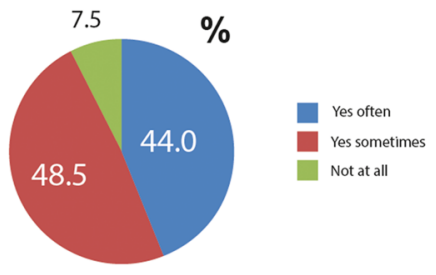

$B$ Do you think that the six main subcategories of T2DM patients identified by AMD algorithms are correct?

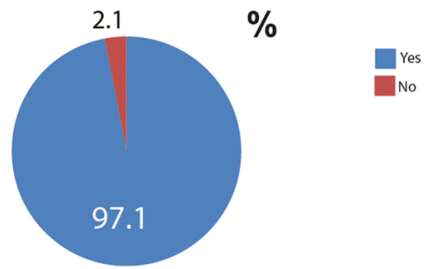

C Do you think that the phenotypization of T2DM patients according to the type and prevalence of their blood sugar levels (fasting, pre-, and post-prandial) can be relevant for choosing the most appropriate therapy?

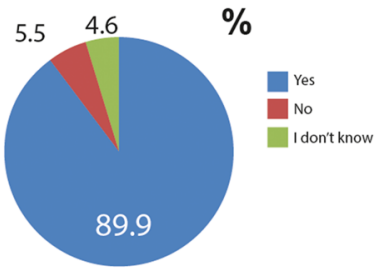

D What did you like most of the AMD algorithms?

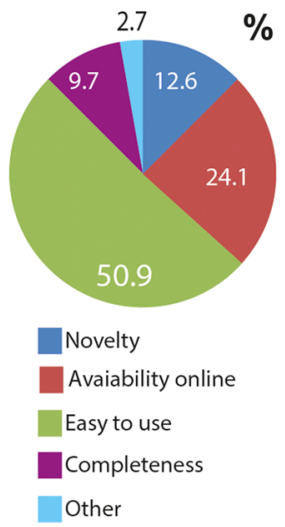

E Do you think that the algorithms are a useful tool for:

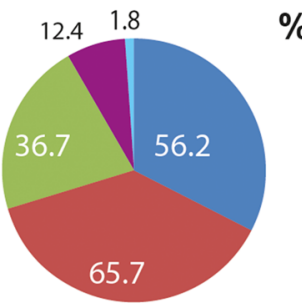

General Practitioners

Diabetologists/ Endocrinologist

Internists

Other specialties

None 
Table 1 Characteristics of participants who believe in the usefulness of the algorithms $(n=418)$

\begin{tabular}{|c|c|c|c|c|c|c|c|c|c|c|}
\hline & \multicolumn{2}{|c|}{ Age category } & \multicolumn{2}{|l|}{ Gender } & \multicolumn{2}{|l|}{ Country } & \multicolumn{2}{|c|}{ Patients monthly } & \multicolumn{2}{|l|}{ Practice } \\
\hline & $45-55$ & $>55$ & Male & Female & Italy & Others & $50-200$ & $>200$ & Hospital & Private \\
\hline $\begin{array}{l}\text { Specialists diabetes/ } \\
\text { endocrinology } \\
(n=320) n(\%)\end{array}$ & $161(50)$ & $159(50)$ & $186(58)$ & $134(42)$ & $294(92)$ & $26(8)$ & $168(53)$ & $152(47)$ & $276(86)$ & $44(14)$ \\
\hline $\begin{array}{l}\text { General } \\
\text { practitioners }(n=37) \\
n(\%)\end{array}$ & $28(75)$ & $19(25)$ & $25(67)$ & $12(33)$ & $29(78)$ & $8(22)$ & $34(92)$ & $3(8)$ & NA & \\
\hline Others $(n=61) n(\%)$ & $36(59)$ & $25(41)$ & $37(60)$ & $24(40)$ & $37(60)$ & $24(40)$ & $41(67)$ & $20(33)$ & $46(75)$ & $15(25)$ \\
\hline
\end{tabular}

GPs, compared to diabetes specialists, considered the algorithms useful for general practice, whereas algorithms were considered useful for specialist practice by diabetes specialists at a greater extent than by GPs $(p<0.01)$. Furthermore, responders from Italy were more likely to consider the algorithms useful for diabetes specialists, whereas those from other countries found them more useful for GPs (both $\mathrm{p}<0.01$ ). In addition, a significantly greater proportion of responders among those aged more than 55 years than among those who were younger found the algorithms useful for diabetes specialists $(p<0.01)$. For greater detail, see table 1 .

At multivariate analysis (stepwise logistic regression), profession and country of origin resulted independently associated with the opinion about the usefulness of algorithms for either GPs and diabetes specialists. Responders from Italy perceived the algorithms more useful for specialists and less useful for GPs, in comparison with participants from other countries, independent of profession. In addition, in comparison with diabetes specialists, GPs considered the algorithm more useful for general practice, and less useful for specialists, independent of country of origin (see table 2).

The vast majority (ie, $97.1 \%$ ) of responders agreed with the six main subcategories of patients with T2DM identified by AMD algorithms (figure 2B). In addition, $89.9 \%$ believed that the phenotypization of patients with T2DM according to their prevalent pattern of hyperglycemia (fasting, preprandial, and postprandial) is useful for choosing the most appropriate antidiabetic therapy (figure $2 \mathrm{C}$ ).
With regard to the question "What did you like most of the AMD algorithms?", half of responders (50.9\%) ranked the simplicity of use in the first place (figure 2D).

Thirty-four $(7.5 \%)$ of the responders considered the algorithms useless; of those, $29(85 \%)$ were diabetologists/endocrinologist, and the great majority of them (28, ie, $82 \%)$ were from Italy.

\section{Online visibility of the algorithms}

In the online survey period and in the months after its conclusion, we registered a significant increase in the number of unique users who visited the websites of the AMD algorithms (http://www.aemmedi.it/algoritmi_it_ 2013 and http://www.aemmedi.it/algoritmi_en_2013/), as well as in the number of page views of the algorithms, and in the average time spent by visitors on algorithms pages, compared to the period preceding the survey (table 3). This increase concerned both Italian users (with an increase of over 10 times of page views in a month) and those from other countries (with an increase of over 50 times). The increase in visitors, number of pages and time spent on algorithms pages was maintained for approximately 6 months after the publication of the survey online, probably to an increased interest promoted by the same survey.

\section{DISCUSSION}

In recent years there has been increasing interest in the issue of treatment personalization, also in the context of diabetes. Personalized medicine emphasizes

Table 2 Characteristics of participants according to their opinion on the usefulness of the algorithms, at multivariate analysis

\begin{tabular}{|c|c|c|c|c|}
\hline \multirow[b]{2}{*}{ Characteristics } & \multicolumn{2}{|l|}{ Useful for GPs } & \multicolumn{2}{|c|}{ Useful for diabetes specialists } \\
\hline & OR (95\% Cl) & p Value & OR (95\% Cl) & p Value \\
\hline Country (Other vs Italy) & 3.87 (1.94 to 7.72$)$ & $<0.001$ & $0.30(0.17$ to 0.55$)$ & $<0.001$ \\
\hline \multicolumn{5}{|l|}{ Profession } \\
\hline Diabetologist & Ref. & - & Ref. & - \\
\hline GP & 6.83 (2.12 to 22.03$)$ & 0.001 & $0.26(0.11$ to 0.64$)$ & 0.003 \\
\hline Other & 0.95 (0.53 to 1.69$)$ & 0.86 & $1.48(0.83$ to 2.64$)$ & 0.18 \\
\hline
\end{tabular}

GP, general practitioner. 
Table 3 Single accesses to the AMD algorithms websites and average time on site before and after the online advertising initiative (http://www.aemmedi.it/algoritmi_it 2013/ and http://www.aemmedi.it/algoritmi_en_2013/)

\begin{tabular}{|c|c|c|c|c|}
\hline \multirow[b]{2}{*}{ Month } & \multirow{2}{*}{$\begin{array}{l}\text { Unique } \\
\text { visitors }\end{array}$} & \multicolumn{2}{|c|}{ Page views } & \multirow{2}{*}{$\begin{array}{l}\text { Average } \\
\text { time on } \\
\text { site }\end{array}$} \\
\hline & & Italian & English & \\
\hline Oct 2013 & 836 & 1971 & 298 & $4^{\prime} 52^{\prime \prime}$ \\
\hline Nov 2013 & 1235 & 4056 & 789 & $8^{\prime} 56^{\prime \prime}$ \\
\hline $\begin{array}{l}\text { Dec } 2013 \\
\text { (advertising) }\end{array}$ & 4726 & 6046 & 11736 & $12^{\prime} 24^{\prime \prime}$ \\
\hline Jan 2014 & 8978 & 9897 & 14054 & $12^{\prime} 44^{\prime \prime}$ \\
\hline Feb 2014 & 9912 & 17613 & 16827 & $13^{\prime} 56^{\prime \prime}$ \\
\hline Mar 2014 & 11927 & 20582 & 14418 & $14^{\prime} 21^{\prime \prime}$ \\
\hline Apr 2014 & 9596 & 16576 & 9026 & $11^{\prime} 27^{\prime \prime}$ \\
\hline May 2014 & 4467 & 11347 & 1263 & $8^{\prime} 56^{\prime \prime}$ \\
\hline Jun 2014 & 3189 & 10921 & 854 & $6^{\prime} 29^{\prime \prime}$ \\
\hline Jul 2014 & 2027 & 7625 & 1278 & $5^{\prime} 31^{\prime \prime}$ \\
\hline Aug 2014 & 926 & 2398 & 981 & $4^{\prime} 08^{\prime \prime}$ \\
\hline Sep 2014 & 1857 & 6538 & 258 & $4^{\prime} 41^{\prime \prime}$ \\
\hline Oct 2014 & 2154 & 11528 & 136 & $7^{\prime} 17^{\prime \prime}$ \\
\hline Nov 2014 & 3127 & 10327 & 318 & $8^{\prime} 02^{\prime \prime}$ \\
\hline Dec 2014 & 2654 & 6428 & 278 & $5^{\prime} 11^{\prime \prime}$ \\
\hline \multirow{2}{*}{ Jan 2015} & 1938 & 5238 & 326 & $4^{\prime} 29^{\prime \prime}$ \\
\hline & 69549 & 149091 & 7284 & $8^{\prime} 13^{\prime \prime}$ \\
\hline
\end{tabular}

AMD, Italian Association of Diabetologists.

the customization of healthcare, with all decisions and practices being tailored to individual patients whenever possible. ${ }^{12}$ Its prerequisite is patient phenotyping, as well as taking the realities of each individual patient's circumstances into account. Its final aim is to optimize therapeutic responses, while improving tolerability and compliance at the same time. It is noteworthy that, in clinical practice, physicians feel more comfortable in pursuing these goals when having pragmatic aids, such as predefined algorithms, are available. ${ }^{13}$

Since 2010, the AMD algorithms have been reviewed and updated three times. The last update in September 2014, as well as the previous one, was shared with the Italian College of General Practitioners (SIMG, Italian Society of General Medicine) in order to adopt a common methodological approach during the medical decision-making process, and included newly approved drugs or expanded indications for existing ones in the European Union.

The main purpose of this online survey was to collect participants' opinion on the AMD algorithms, to assess their applicability in clinical practice and to get suggestions for their implementation and for future versions. The other aim of our initiative was to increase the visibility of the websites of the algorithms, especially for non-Italian speaking users.

At our knowledge, this is the first online survey on a web-based innovative personalized algorithm for the treatment of T2DM. Although the results of the survey show wide agreement by responders on the utility of the algorithm proposed, we understand that it does not cover all possible needs of patients with T2DM for a personalized treatment. This will be an incentive for expanding the algorithm in the next future, taking into account more different phenotypes such us patients with previous cardiovascular events, gestational diabetes and for approaching major cardiovascular risk factors associated with T2DM such as arterial hypertension and dyslipidemia. Only thirty-four $(7.5 \%)$ responders did not find the algorithm of any utility, even if many of them found it well designed or useful for GPs or specialists.

With regard to the number of responders, it must be acknowledged that participation in the survey did not provide any form of compensation, unlike many other surveys received daily by each physician. The participation of Italian doctors, much higher than that of responders from other countries, could depend on a more profound knowledge of the algorithms. The fact that AMD repeatedly solicited via email the participation of its members in the survey could be another explanation for this finding. Furthermore, the advertising for Italian physicians continued from December 2013 to September 2014, while online advertising on the journal home page lasted only a month.

The opinion of responders from Italy in perceiving the algorithms more useful for specialists and less useful for GPs, in comparison with participants from other countries, may be the consequence of national peculiarities in the organization of diabetes care. All Italian citizens, regardless of social class or income, are cared for by a general practitioner as part of the National Health System. GPs cooperate with a public network of about 700 diabetes outpatient clinics in providing primary care for persons with diabetes, which is completely free of charge. ${ }^{14-16}$ Italian GPs can prescribe the most innovative drugs only on specialists' authorization, and therefore, GPs who do not keep in touch with diabetes care teams cannot autonomously decide to use GLP-1 receptor agonists and DDP4 inhibitors. Generally, GPs are more involved in earlier stages of the natural history of diabetes, whereas diabetes specialists concentrate most of their resources on inadequately controlled, insulin-treated or complicated patients. For example, insulin therapy is initiated by specialists in most instances. ${ }^{17}$ On the other hand, current rules for reimbursement establish that 'innovative' drugs (which include DPP4 inhibitors and GLP-1 receptor agonists) are provided free of charge only when prescribed by a certified specialist. As a consequence, available therapeutic choices for Italian GPs are limited to older drugs: this could explain the perceived reduced usefulness of the algorithms for this medical category. Conversely, in other countries, where all physicians have access to all therapies, GPs could benefit from the use of algorithms more than diabetes specialists.

Limitations of the study must to be acknowledged. The study design implies that the results of the survey reflect the opinions of its participants; they could be systematically different from the opinions of non- 
responders. Nevertheless, the quite large number of physicians involved from different countries seems reassuring regarding the generalizability of the results. One more limit to be considered is that the vast majority of respondents are Italians and this could influence the results. We also need to take into account that treatment algorithms from other organizations are more extensive than ours and this could represent a possible bias for those respondents who have more familiarity with such algorithms.

Patients with T2DM are heterogeneous in their clinical features, and there is a clinical need for personalized algorithms that cover several issues. In our view tailored therapy is the best approach to optimize diabetes care, and the Italian algorithm for the treatment of T2DM is an innovative, accessible and easy to use tool for every day application and for every clinician who manage patients with diabetes. Even if we are fully aware that AMD algorithms cannot cover all of the possible combinations encountered in daily clinical practice, we hope that they may counteract clinical inertia, contributing, in a small way, to improve the approach to a personalized management of T2DM.

\section{Author affiliations}

${ }^{1}$ Department of Oncological Endocrinology, AOU Città della Salute e della Scienza-Molinette, Turin, Italy

${ }^{2}$ Department of Diabetes Agency, Careggi Teaching Hospital, Florence, Italy

${ }^{3}$ Unit of Internal Medicine, IRCCS Casa Sollievo della Sofferenza, San

Giovanni Rotondo, Italy

${ }^{4}$ Department of Clinical and Experimental Medicine, Second University of

Naples, Naples, Italy

${ }^{5}$ Diabetes Center, A.S.S. 1 Triestina, Trieste, Italy

${ }^{6}$ Ligurian Health Agency, Genoa, Italy

${ }^{7}$ Department of Internal Medicine, University of Messina, Messina, Italy

${ }^{8}$ Department of Cardiovascular and Metabolic Diseases, Istituto di Ricovero e

Cura a Carattere Scientifico Gruppo Multimedica, Sesto San Giovanni, Italy

${ }^{9}$ Italian College of General Practitioners (Società Italiana di Medicina Generale), Florence, Italy

${ }^{10}$ Department of Endocrinology, Hospital Clinic de Barcelona, Institut d'Investigacions Biomèdiques August Pi i Sunyer (IDIBAPS), Barcelona, Spain

${ }^{11}$ Centro de Investigacion Biomèdica en Red de Diabetes y Enfermedades

Metabolicas Asociadas (CIBERDEM), Barcelona, Spain

\section{Collaborators On behalf of Associazione Medici Diabetologi (AMD)}

Contributors MG, ADM, RC and AC designed the survey. MG, SDC and SG wrote initial draft of manuscript. EM performed the analysis. MG reviewed and edited the manuscript. All authors contributed to the interpretation of the data and to the critical revision of the manuscript for important intellectual content: All authors have read and approved the final manuscript. MG, EM and $A C$ are the guarantors of this work and, as such, had full access to all the data in the study and take responsibility for the integrity of the data and the accuracy of the data analysis. The authors would like to express their gratitude to Giorgio Mantovani by Infomedica for collecting data, and to Luisa Brunetti for her linguistic assistance.

Funding This work was self-supported by the Italian Association of Diabetologists (Associazione Medici Diabetologi, AMD). MG takes full responsibility for the work as a whole, including the study design, access to data and the decision to submit and publish the manuscript.

Competing interests None declared.

Provenance and peer review Not commissioned; externally peer reviewed.
Data sharing statement The database of survey's participants answers for this research will be available on request.

Open Access This is an Open Access article distributed in accordance with the Creative Commons Attribution Non Commercial (CC BY-NC 4.0) license, which permits others to distribute, remix, adapt, build upon this work noncommercially, and license their derivative works on different terms, provided the original work is properly cited and the use is non-commercial. See: http:// creativecommons.org/licenses/by-nc/4.0/

\section{REFERENCES}

1. Inzucchi SE, Bergenstal RM, Buse JB, et al. Management of hyperglycemia in type 2 diabetes, 2015: a patient-centered approach: update to a position statement of the American Diabetes Association and the European Association for the Study of Diabetes. Diabetes Care 2015;38:140-9.

2. International Diabetes Federation Clinical Guidelines Task Force. Global Guideline for Type 2 Diabetes, 2012. http://www.idf.org/ global-guideline-type-2-diabetes-2012 (accessed 24 Feb 2015).

3. Garber AJ, Abrahamson MJ, Barzilay JI, et al. American Association of Clinical Endocrinologists' comprehensive diabetes management algorithm 2013 consensus statement-executive summary. Endocr Pract 2013;19:536-57.

4. Skyler JS, Bergenstal R, Bonow RO, et al, American Diabetes Association; American College of Cardiology Foundation; American Heart Association. Intensive glycemic control and the prevention of cardiovascular events: implications of the ACCORD, ADVANCE, and VA diabetes trials: a position statement of the American Diabetes Association and a scientific statement of the American College of Cardiology Foundation and the American Heart Association. Diabetes Care 2009;32:187-92.

5. Diabetes treatment algorithm from the Diabetes Current Care Guideline Working group set up by the Finnish Medical Society Duodecim and the Finnish Society of Internal Medicine. 2009. http:// www.terveysportti.fi/xmedia/ccs/varhainen_diabetes_en.html (accessed 24 Feb 2015).

6. Virkamäki A, Saltevo J. Finnish Current Care Guideline for Diabetes: interactive approach to improve individualised treatment. Diabetologia 2011;54:1264-5.

7. Ceriello A, Gallo M, Gentile S, et al, on behalf of Associazione Medici Diabetologi (AMD). To what extent is the new position statement of the American Diabetes Association (ADA) and the European Association for the Study of Diabetes (EASD) 'personalised'? Diabetologia 2012;55:2853-5.

8. International Diabetes Federation. Guideline on self-monitoring of blood glucose in non-insulin treated type 2 diabetes. 2009. http:// www.idf.org/guidelines/self-monitoring (accessed 24 Feb 2015).

9. Ceriello A, Gallo M, Armentano V, et al, on behalf of the Associazione Medici Diabetologi. Personalizing treatment in type 2 diabetes: a self-monitoring of blood glucose inclusive innovative approach. Diabetes Technol Ther 2012;14:373-8.

10. Esposito K, Gentile S, Candido R, et al, Associazione Medici Diabetologi. Management of hyperglycemia in type 2 diabetes: evidence and uncertainty. Cardiovasc Diabetol 2013;12:81.

11. Italian Association of Medical Diabetologists AMD. Personalisation of therapy in type 2 diabetes. http://www.aemmedi.it/algoritmi en 2014/ (accessed 24 Feb 2015)

12. Goldberger JJ, Buxton AE. Personalized medicine vs guideline-based medicine. JAMA 2013;309:2559-260.

13. Stone TT, Kivlahan $\mathrm{CH}$, Cox KR. Evaluation of physician preferences for guideline implementation. Am J Med Qual 1999;14:170-7.

14. Rossi MC, Nicolucci A, Arcangeli A, et al, Associazione Medici Diabetologi Annals Study Group. Baseline quality-of-care data from a quality-improvement program implemented by a network of diabetes outpatient clinics. Diabetes Care 2008;31:2166-8.

15. Nicolucci A, Rossi MC, Arcangeli A, et al, AMD-Annals Study Group. Four-year impact of a continuous quality improvement effort implemented by a network of diabetes outpatient clinics: the AMD-Annals initiative. Diabet Med 2010;27:1041-8.

16. Rossi MC, Lucisano G, Comaschi M, et al, AMD-QUASAR Study Group. Quality of diabetes care predicts the development of cardiovascular events: results of the AMD-QUASAR. Diabetes Care 2011;34:347-52.

17. Strollo F, Guarino G, Marino G, et al. Different prevalence of metabolic control and chronic complication rate according to the time of referral to a diabetes care unit in the elderly. Acta Diabetol 2014:51:447-53. 